\title{
Association of Circulating Neuregulin-4 with Presence and Severity of Coronary Artery Disease
}

\author{
Qing-Ping Tian, ${ }^{1} \mathrm{MD}$, Mei-Lin Liu, ${ }^{1} \mathrm{MD}$, Chao-Shu Tang, ${ }^{2} \mathrm{PhD}$, Lin Xue, ${ }^{3}$, \\ Yong-Zheng Pang, ${ }^{2}$ and Yong-Fen $\mathrm{Qi},{ }^{4} \mathrm{PhD}$
}

\begin{abstract}
Summary
Neuregulin-4 (Nrg4) is a newly discovered adipokine that is synthesized in many tissues and plays an important role in modulating systemic energy metabolism and in the development of metabolic disorders. However, little is known about the relationship between Nrg4 and coronary artery disease (CAD). In this study, we investigated the association between $\mathrm{Nrg} 4$ and the presence and severity of CAD.

We enrolled 73 patients diagnosed by coronary angiography (CAG) as having CAD and 32 controls. The CAD group was divided into two subgroups according to their SYNTAX score. Plasma levels of Nrg4 were measured in all participants and compared among different groups. The relationship between Nrg4 and CAD was analyzed. Receiver operating characteristic (ROC) analysis was conducted to evaluate the usefulness Nrg4 in assessing the presence and severity of CAD.

Nrg4 levels were negatively associated with the SYNTAX score $(r=-0.401, P=0.000)$. The patients with a higher SYNTAX score had significantly lower Nrg4 levels as compared with the low SYNTAX score subgroup and the controls $(P<0.05)$. The Nrg4 levels of the low SYNTAX score subgroup were much lower than controls $(P<0.05)$. Furthermore, an association between Nrg4 and CAD (odds ratio, 0.279; 95\% confidence interval, $0.088-0.882$ ) was observed. Nrg4 had $43.8 \%$ sensitivity and $96.9 \%$ specificity for identifying CAD, and $73.1 \%$ sensitivity and $87.3 \%$ specificity for identifying patients who had severe coronary artery lesions.
\end{abstract}

$\mathrm{Nrg} 4$ levels were found to be inversely associated with the presence and severity of CAD.

Key words: Adipokine, SYNTAX score

(Int Heart J 2019; 60: 45-49)

$\mathrm{N}$ euregulins (NRGs) are signaling proteins which mediate cell-cell interactions and influence the proliferation, apoptosis, migration, differentiation, and adhesion of cells in many kinds of tissues. ${ }^{1-4)}$ Neuregulin-4 (Nrg4) is a secreted adipokine of this epidermal growth factor-like (EGFL) family recently identified as playing an important role in modulating systemic energy metabolism and in the development of metabolic disorders in rodent and human obesity. ${ }^{5,6)}$ Mice overexpressing Nrg4 had inhibited lipogenesis and reduced chronic inflammation, resulting in the prevention of highfat diet induced adiposity and fatty liver and the improvement of insulin sensitivity compared to controls. ${ }^{7)}$ In a case-control study in China, it was shown that the serum Nrg4 level was an independent factor associated with nonalcoholic fatty liver disease (NAFLD) and reported that $\mathrm{Nrg} 4$ might have a protective role in the development of NAFLD. ${ }^{8)}$ Using a rat insulinoma cell line as an in vitro model system, it was found that Nrg4 had a very pronounced effect on insulin secretion.9) A cross-sectional study indicated that circulating $\mathrm{Nrg} 4$ concentrations were inversely associated with the risk of metabolic syndrome in obese adults. ${ }^{5}$

\section{Editorial p.1}

Coronary artery disease (CAD) is a major cause of morbidity and mortality worldwide. ${ }^{10)}$ Identification of risk factors for the development of CAD could aid the development of prevention strategies. Obesity and metabolic disorder are the risk factors of developing CAD. ${ }^{11,12)}$ And decreased insulin sensitivity and low-grade inflammation may largely account for the development of CAD. Previous research has indicated that several cytokines secreted by adipocytes have pro- and anti-inflammatory properties and are associated with the incidence of CAD. ${ }^{5}$

From the ${ }^{1}$ Department of Geriatrics, Peking University First Hospital, Beijing, China, ${ }^{2}$ Institute of Cardiovascular Disease, Peking University First Hospital, Beijing, China, ${ }^{3}$ Department of Cardiology, Peking University First Hospital, Beijing, China and ${ }^{4}$ Key Laboratory of Molecular Cardiovascular Science, Ministry of Education, School of Basic Medical Sciences, Peking University Health Science Center, Beijing, China.

This work was supported by the National Natural Science Foundation of China (Nos. 91339203 and 81670434 to Y.F. Qi).

Address for correspondence: Qing-Ping Tian, MD, Department of Geriatrics, Peking University First Hospital, No8. Xishiku Street, Xicheng District, Beijing 100034, China. E-mail: tianqingping@bjmu.edu.cn or 13621267654@163.com or Yong-Fen Qi, PhD, Key Laboratory of Molecular Cardiovascular Science, Ministry of Education, Peking University Health Science Center, Beijing 100083, China. E-mail: yongfenqi@163.com

Received for publication February 28, 2018. Revised and accepted April 16, 2018.

Released in advance online on J-STAGE November 5, 2018.

doi: 10.1536/ihj.18-130

All rights reserved by the International Heart Journal Association. 
Information is not available regarding the association between circulating $\mathrm{Nrg} 4$ and the presence and severity of CAD in human subjects. Only one study showed that circulating Nrg4 concentrations were inversely correlated with carotid intima-media thickness (CIMT), which is a subclinical atherosclerotic disease and can predict cardiovascular disease (CVD) events in the general population. ${ }^{5,13,14)}$ However, the relationship between $\mathrm{Nrg} 4$ and the presence and severity of CAD is unclear. Accordingly, the aim of the current study was to explore this relationship. We measured the plasma levels of $\mathrm{Nrg} 4$ in patients with $\mathrm{CAD}$ and analyzed the correlation of $\mathrm{Nrg} 4$ and degree of coronary stenosis.

\section{Methods}

Subjects: Seventy-three patients who underwent coronary angiography (CAG) and were diagnosed with CAD and 32 patients with normal coronary arteries were enrolled in the study. Patients with previous coronary artery bypass grafting $(\mathrm{CABG})$ and/or acute coronary syndrome were excluded from the study. Patients with diabetes, an acute infection, chronic inflammatory diseases, severe hepatic or renal dysfunction, or malignancy were also excluded. The study was proved by the Ethics Committee of Peking University First Hospital, Beijing, and complied with the principles outlined in the Declaration of Helsinki. Informed consent was obtained from the subjects before their inclusion.

Coronary angiography: Coronary angiography (CAG) was performed using the Judkins technique through the femoral or radial artery. Each coronary artery was displayed in at least two different plane images. The SYNTAX score was used to define the severity of coronary lesions. The SYNTAX score was calculated in all patients by two experienced interventional cardiologists. The SYNTAX score was determined for all coronary lesions with $>$ $50 \%$ diameter stenosis in a vessel $>1.5 \mathrm{~mm}$, based on the SYNTAX score calculator (http://www.syntax.com). The CAD group was divided into two subgroups as the high SYNTAX score ( $\geq 15$ ) subgroup (26 patients) and lower SYNTAX score $(<15)$ subgroup (47 patients).

Clinical examinations: The medical history, blood pressure, smoking history, body mass index (BMI), medications, and comorbidities of the patients were recorded.

Venous blood samples were obtained from the antecubital vein on admission. All patients underwent laboratory tests using standard methods following overnight fasting to determine serum triglycerides (TG), total cholesterol (TCHO), high-density lipoprotein (HDL), lowdensity lipoprotein (LDL), creatinine (Cr), high-sensitivity C-reactive protein (hsCRP), brain natriuretic peptide (BNP), and glycosylated hemoglobin A1c (HbA1C).

Blood samples for $\mathrm{Nrg} 4$ measurements were drawn into tubes containing disodium ethylene diamine tetraacidic acid $(1 \mathrm{mg} / \mathrm{mL})$ and aprotinin $(500 \mathrm{KIU} / \mathrm{mL}$; Sigma, St. Louis, MO, USA), and then immediately centrifuged at $3500 \mathrm{~g}$ for 10 minutes at $4^{\circ} \mathrm{C}$. Plasma was stored at $-80^{\circ} \mathrm{C}$. $\mathrm{Nrg} 4$ was measured using an EIA kit (EK-056-24, Phoenix Pharmaceuticals, USA) as recommended by the manufacturer's protocol. The intra- and inter-assay coefficients of variation were $<5 \%$ and $<$ $14 \%$, respectively. The detection range was 0 to $100 \mathrm{ng} /$ $\mathrm{mL}$.

Statistical analysis: IBM SPSS Statistics version14.0 was used for statistical analysis. Continuous variables are expressed as the mean \pm standard deviation, and categorical variables are expressed as percentages and/or numbers. The chi-square test was used to compare the categorical variables. One-way analysis of variance test was used to compare normally distributed variables between 3 groups. Correlations between variables were tested by Pearson and nonparametric Spearman correlation analysis. A logistic regression model was used to examine the risk factors of CAD. Receiver operating characteristic (ROC) curves were generated and areas under the curve (AUC) were compared to identify the cut-off values of Nrg4 to assess the presence and severity of CAD. Two-sided values of $P$ $<0.05$ were considered statistically significant.

\section{Results}

Clinical characteristics: Gender, BMI, systolic blood pressure (SBP), diastolic blood pressure (DBP), fasting glucose, HbA1C, TG, TCHO, HDL, LDL, use of calcium channel blocker (CCB) and angiotensin converting enzyme inhibitors (ACEI) or angiotensin receptor blocker (ARB), left ventricular ejection fraction (LVEF), and history of hypertension showed no statistically significant differences among the groups. There were more patients in the CAD (either SYNTAX score higher or lower) group using aspirin, $\beta$-blocker, and statins, and more smokers. The levels of hsCRP and BNP of the patients with a higher SYNTAX score were higher than the patients with a lower SYNTAX score. The patients with a higher SYNTAX score were older and had a lower estimated glomerular filtration rate (eGFR) level than the patients with a lower SYNTAX score (Table I).

Circulating Nrg4 concentration: The patients with a higher SYNTAX score had significantly lower Nrg4 levels $(0.87 \pm 0.55 \mathrm{ng} / \mathrm{mL})$ as compared with the low SYNTAX score group $(1.35 \pm 0.63 \mathrm{ng} / \mathrm{mL})$ and control group $(1.41$ $\pm 0.17 \mathrm{ng} / \mathrm{mL}$ ). The Nrg4 levels of the low SYNTAX patients were lower than the control group and the difference was also statistically significant (Table I).

Relationships between variables and presence and severity of CAD: Using the Pearson and nonparametric Spearman correlation tests for all collected variables, it was observed that human circulating $\mathrm{Nrg} 4$ levels were not associated with age, BMI, TG, TCHO, LDL, HDL, HbA1C, or hsCRP, but were negatively associated with the SYNTAX score (Table II).

Age, gender, BMI, hypertension, smoking, TG, LDL, HbA1C, hsCRP, and Nrg4 were used in the logistic regression analysis with a backward stepwise method. It was found that hypertension, LDL, Nrg4, and smoking were significantly correlated with CAD (Table III).

The level of Nrg4 was inversely correlated with the SYNTAX score $(P=0.008$ in nonlinear regression analysis) (Figure 1)

Cut-off values of $\mathrm{Nrg} 4$ in assessing the presence and severity of CAD: The results of ROC curve analysis (Fig- 
Table I. Clinical Characteristics of the Study Participants

\begin{tabular}{|c|c|c|c|c|}
\hline & $\begin{array}{c}\text { Controls } \\
(n=32)\end{array}$ & $\begin{array}{c}\text { SYNTAX score } \\
(<15) \\
(n=47)\end{array}$ & $\begin{array}{c}\text { SYNTAX score } \\
(\geq 15) \\
(n=26)\end{array}$ & $P$ value \\
\hline Age (years) & $62.56 \pm 11.79$ & $61.60 \pm 7.94$ & $67.00 \pm 11.35^{\dagger}$ & 0.088 \\
\hline Gender (male) (\%) & $18(56.25)$ & $34(72.34)$ & $18(69.23)$ & 0.313 \\
\hline BMI $\left(\mathrm{kg} / \mathrm{m}^{2}\right)$ & $24.14 \pm 6.02$ & $25.13 \pm 5.25$ & $24.84 \pm 7.97$ & 0.783 \\
\hline Smoking (\%) & $11(34.38)$ & $35(74.47)^{*}$ & $17(65.38)^{*}$ & 0.001 \\
\hline $\mathrm{SBP}(\mathrm{mmHg})$ & $128.44 \pm 15.70$ & $134.34 \pm 16.94$ & $138.96 \pm 17.63$ & 0.521 \\
\hline DBP (mmHg) & $73.38 \pm 11.81$ & $78.77 \pm 13.04$ & $77.00 \pm 9.06$ & 0.090 \\
\hline Hypertension (\%) & $17(53.13)$ & $28(59.58)$ & $16(61.54)$ & 0.494 \\
\hline Fasting glucose $(\mathrm{mmol} / \mathrm{L})$ & $5.27 \pm 0.96$ & $5.49 \pm 0.98$ & $5.71 \pm 1.02$ & 0.263 \\
\hline $\operatorname{HbA1C}(\%)$ & $5.96 \pm 0.35$ & $6.01 \pm 0.49$ & $5.94 \pm 0.52$ & 0.810 \\
\hline $\mathrm{TG}(\mathrm{mmol} / \mathrm{L})$ & $1.55 \pm 0.82$ & $1.72 \pm 1.02$ & $1.37 \pm 0.50$ & 0.236 \\
\hline $\mathrm{TCHO}(\mathrm{mmol} / \mathrm{L})$ & $4.08 \pm 0.83$ & $4.27 \pm 0.98$ & $4.24 \pm 0.66$ & 0.633 \\
\hline $\mathrm{HDL}(\mathrm{mmol} / \mathrm{L})$ & $1.14 \pm 0.33$ & $1.07 \pm 0.24$ & $1.02 \pm 0.30$ & 0.302 \\
\hline $\mathrm{LDL}(\mathrm{mmol} / \mathrm{L})$ & $2.28 \pm 0.63$ & $2.53 \pm 0.88$ & $2.64 \pm 0.61$ & 0.167 \\
\hline hsCRP (mg/L) & $3.08 \pm 9.10$ & $3.06 \pm 4.22$ & $7.37 \pm 11.33^{* \dagger}$ & 0.068 \\
\hline eGFR $\left(\mathrm{mL} / \mathrm{minute} / 1.73 \mathrm{~m}^{2}\right)$ & $81.59 \pm 14.65$ & $80.50 \pm 16.60$ & $66.76 \pm 17.72 * \dagger$ & 0.001 \\
\hline $\mathrm{BNP}(\mathrm{pg} / \mathrm{mL})$ & $117.81 \pm 175.82$ & $80.09 \pm 93.02$ & $173.76 \pm 162.03^{\dagger}$ & 0.033 \\
\hline $\mathrm{Nrg} 4(\mathrm{ng} / \mathrm{mL})$ & $1.41 \pm 0.17$ & $1.35 \pm 0.63^{*}$ & $0.87 \pm 0.55^{* \dagger}$ & 0.000 \\
\hline Aspirin (\%) & $20(62.50)$ & $46(97.87)^{*}$ & $26(100)^{*}$ & 0.000 \\
\hline$\beta$-blocker (\%) & $14(43.75)$ & $32(68.08)^{*}$ & $21(80.77)^{*}$ & 0.010 \\
\hline Statins $(\%)$ & $24(75)$ & $46(97.87)^{*}$ & $26(100)^{*}$ & 0.000 \\
\hline ACEI or ARB (\%) & $17(53.13)$ & $23(48.94)$ & $13(50)$ & 0.934 \\
\hline CCB $(\%)$ & $7(21.88)$ & $12(25.53)$ & $6(23.08)$ & 0.927 \\
\hline $\operatorname{LVEF}(\%)$ & $65.21 \pm 13.48$ & $66.89 \pm 8.81$ & $66.37 \pm 10.29$ & 0.803 \\
\hline
\end{tabular}

Data are presented as the mean $\pm \mathrm{SD}$ or $n(\%) . * P<0.05$ compared with control; ${ }^{\dagger} P<0.05$ compared with the SYNTAX score $(<15)$. BMI indicates body mass index; SBP, systolic blood pressure; DBP, diastolic blood pressure; HbA1C, glycosylated hemoglobin A1c; TG, triglycerides; TCHO, total cholesterol; HDL, high-density lipoprotein; LDL, lowdensity lipoprotein; hsCRP; high-sensitivity C-reactive protein; eGFR, estimated glomerular filtration rate; BNP, brain natriuretic peptide; $\mathrm{Nrg} 4$, neuregulin-4; ACEI, angiotensin converting enzyme inhibitor; ARB, angiotensin receptor blocker; CCB, calcium channel blocker; and LVEF, left ventricular ejection fraction.

Table II. Variables Associated with Nrg4 in All Subjects

\begin{tabular}{lcc}
\hline Variable & $\begin{array}{c}\text { Correlation } \\
\text { coefficient }\end{array}$ & $P$ value \\
\hline Age & -0.070 & 0.477 \\
BMI & 0.19 & 0.849 \\
TG & 0.275 & 0.055 \\
TCHO & 0.127 & 0.205 \\
LDL & 0.073 & 0.459 \\
HDL & -0.025 & 0.808 \\
hsCRP & -0.043 & 0.666 \\
HbA1C & 0.041 & 0.689 \\
SYNTAX & -0.401 & 0.000 \\
\hline
\end{tabular}

BMI indicates body mass index; TG, triglycerides; TCHO, total cholesterol; LDL, lowdensity lipoprotein; HDL, high-density lipoprotein; hsCRP; high-sensitivity C-reactive protein; and $\mathrm{HbA1C}$, glycosylated hemoglobin Alc.

ure 2) revealed the AUC of $\mathrm{Nrg} 4$ in assessing the presence and severity of CAD. The AUC were 0.629 and 0.802 , respectively. Nrg4 had $43.8 \%$ sensitivity and $96.9 \%$ specificity for identifying CAD, and $73.1 \%$ sensitivity and $87.3 \%$ specificity for identifying patients who had severe coronary artery lesions.

\section{Discussion}

In the present study, we reported for the first time that circulating Nrg4 concentrations were significantly reduced in patients with CAD and Nrg4 levels were lower in the patients with a higher SYNTAX score. These findings indicated that $\mathrm{Nrg} 4$ levels were inversely associated with the presence and severity of CAD. Nrg4 might be a protective factor in cardiovascular diseases.

It has been established that adipose tissue is a highly dynamic endocrine organ with central roles in the regulation of energy metabolism. Brown adipocytes dissipate energy, whereas white adipocytes are an energy storage site. Activation of brown adipose tissue (BAT) increases energy expenditure. ${ }^{15)}$ Inducing brown-like adipocyte formation in white adipose tissue (WAT) may be valuable to ameliorate arterial stiffness. ${ }^{16}$ ) Nrg4 was recently identified as a brown-fat-enriched endocrine factor. It could be a key factor for the development of BAT and acquisition of BAT features of WAT depots by promoting the growth of neurites and thus enhancing innervation of the tissues needed to activate the thermogenic functions. $\left.{ }^{6}\right) \mathrm{Nrg} 4$ has provided new insight into the prevention of obesity associated disorders. Wang, et $a l^{17)}$ reported that $\mathrm{Nrg} 4$ expression was reduced in murine and human obesity and Nrg4 transgenic mice had lower blood glucose and improved glucose tolerance and insulin sensitivity. Dai, et $a l^{8)}$ reported that 
Table III. Logistic Regression Analysis for the Presence of CAD

\begin{tabular}{lcccl}
\hline & B & Wals & Sig & \multicolumn{1}{c}{ OR (95\%CI) } \\
\hline Hypertension $n(\%)$ & 1.351 & 5.163 & 0.023 & $3.861(1.204-12.383)$ \\
LDL (mmol/L) & 1.207 & 6.323 & 0.012 & $3.344(1.305-8.569)$ \\
Nrg4 (ng/mL) & -1.278 & 4.725 & 0.030 & $0.279(0.088-0.882)$ \\
Smoking $n(\%)$ & 1.827 & 8.467 & 0.004 & $6.215(1.816-21.279)$ \\
\hline
\end{tabular}

B indicates regression coefficient; Wals, Wald test statistics; and Sig, significance.

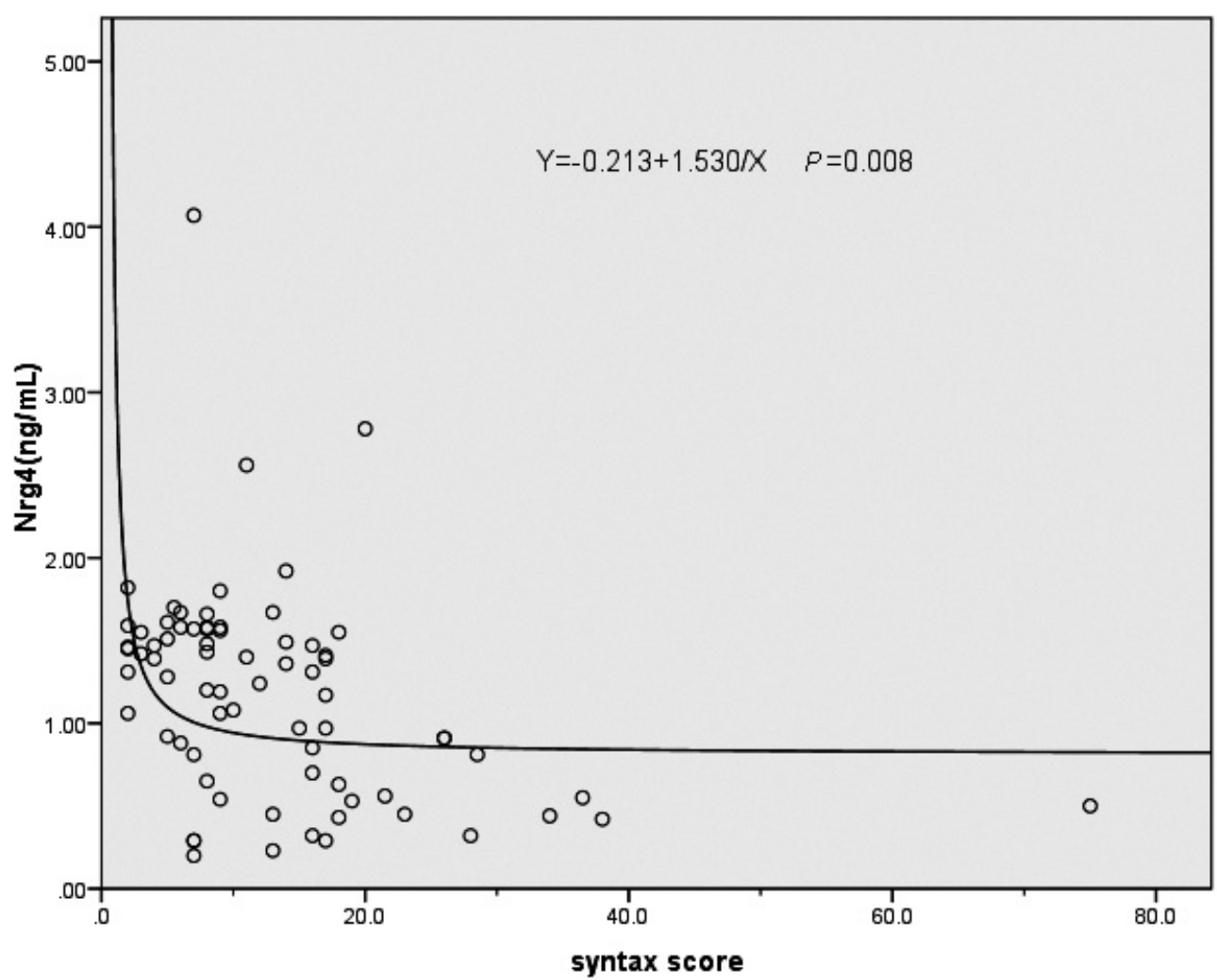

Figure 1. Inverse correlation between the SYNTAX score and $\operatorname{Nrg} 4(P=0.008$ in nonlinear regression analysis $)$. The approximate equation is: $\mathrm{Y}=-0.213+1.530 / \mathrm{X}$.

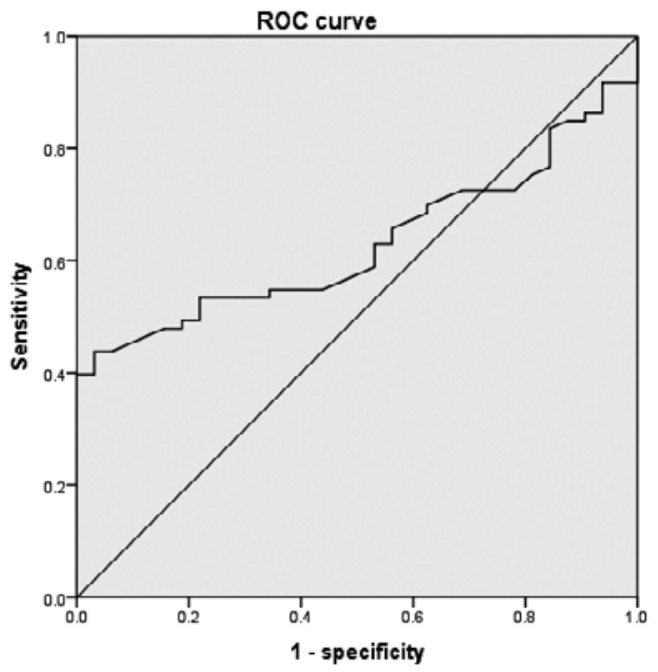

Diagnal segments are produced by ties

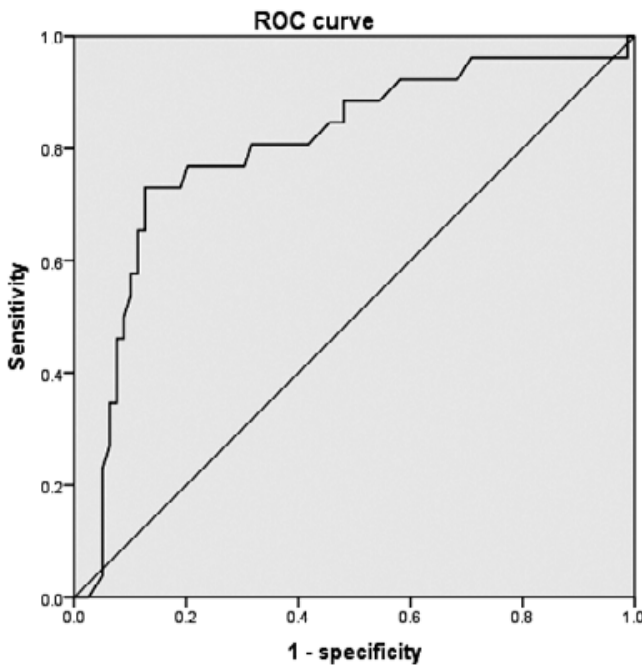

Diagnal segments are produced by ties

Figure 2. ROC analysis results for $\mathrm{Nrg} 4$ in assessing the presence and severity of CAD. 
Nrg4 levels were decreased in NAFLD subjects versus non-NAFLD controls. Cai, et al. ${ }^{5)}$ provided new evidence that $\mathrm{Nrg} 4$ was inversely associated with hepatic fat content in obese adults. All of these results suggest that Nrg4 may be involved in crosstalk between BAT and obesity associated disorders.

Obesity is associated with cardiovascular risk factors and increased risk of CAD. Jiang, et $a l^{18)}$ found that circulating Nrg4 was inversely associated with CIMT, which was the subclinical atherosclerotic disease and can predict $\mathrm{CAD}$ events in the general population. In this study, we found that lower $\mathrm{Nrg} 4$ concentrations were correlated with the presence of CAD and Nrg4 levels were negatively associated with the SYNTAX score. Furthermore, adjustment for age, gender, BMI, and $\mathrm{HbA} 1 \mathrm{C}$ did not attenuate this association. These findings indicated that low circulating Nrg4 could be a new protective adipokine against CAD.

Little is known about the role of Nrg4 in the pathogenesis of CAD. The possible protective mechanism may be a direct or indirect connection between this molecule and the cardiovascular system. Jiang, et $a l^{18)}$ found that Nrg4 may protect against CAD via mechanisms independent of improving insulin resistance and obesity. $\mathrm{Nrg} 4$, as an autocrine, paracrine, or endocrine factor, can activate tyrosine kinases Erb3 and Erb4 in the cardiovascular system, which are important transcriptional regulators of cardiomyocyte proliferation and coordinate glucose and lipid metabolism with thermogenesis. ${ }^{19-22)}$ However, as a newly discovered factor, the exact effect of Nrg4 in CAD needs to be further studied in vivo and in vitro.

Our study provided for the first time evidence that circulating Nrg4 concentrations were significantly reduced in patients with CAD and Nrg4 levels were inversely associated with the presence and severity of CAD. Nrg4 might be a new protective adipokine of CAD. Results of ROC curve analysis revealed that measuring Nrg4 may be more useful for identifying patients with high SYNTAX scores than identifying the presence of CAD. However, there are several limitations in the study. First, the study was based on data with a relatively limited sample size. Second, given its cross-sectional design, the causal relationship between circulating $\mathrm{Nrg} 4$ and CAD could not be determined. Further large prospective studies will be needed to elucidate the exact relationship between $\mathrm{Nrg} 4$ and $\mathrm{CAD}$.

\section{Disclosures}

Conflicts of interest: None.

\section{References}

1. Falls DL. Neuregulins: functions, forms, and signaling strategies. Exp Cell Res 2003; 284: 14-30.

2. Christian M. Transcriptional fingerprinting of "browning" white fat identifies NRG4 as a novel adipokine. Adipocyte 2014; 4
$50-4$

3. Harari D, Tzahar E, Romano J, et al. Neuregulin-4: a novel growth factor that acts through the ErbB-4 receptor tyrosine kinase. Oncogene 1999; 18: 2681-9.

4. Hobbs SS, Coffing SL, Le AT, et al. Neuregulin isoforms exhibit distinct patterns of ErbB family receptor activation. Oncogene 2002; 21: 8442-52.

5. Cai C, Lin M, Xu Y, Li X, Yang S, Zhang H. Association of circulating neuregulin 4 with metabolic syndrome in obese adults: a cross-sectional study. BMC Med 2016; 14: 165.

6. Rosell M, Kaforou M, Frontini A, et al. Brown and white adipose tissues: intrinsic differences in gene expression and response to cold exposure in mice. Am J Physiol Endocrinol Metab 2014; 306: E945-64.

7. Ma Y, Gao M, Liu D. Preventing high fat diet-induced obesity and improving insulin sensitivity through neuregulin 4 gene transfer. Sci Rep 2016; 6: 26242.

8. Dai YN, Zhu JZ, Fang ZY, et al. A case-control study: Association between serum neuregulin 4 level and non-alcoholic fatty liver disease. Metabolism 2015; 64: 1667-73.

9. South JC, Blackburn E, Brown IR, Gullick WJ. The neuregulin system of ligands and their receptors in rat islets of Langerhans. Endocrinology 2013; 154: 2385-92.

10. Smith SC Jr. Screening for high-risk cardiovascular disease: a challenge for the guidelines: comment on "systematic review of guidelines on cardiovascular risk assessment: which recommendations should clinicians follow for a cardiovascular health check?". Arch Intern Med 2010; 170: 40-2.

11. Naito R, Miyauchi K. Coronary artery disease and type 2 diabetes mellitus. Int Heart J 2017; 58: 475-80.

12. Zeller J, Strack C, Fenk S, et al. Relation between obesity, metabolic syndrome, successful long-term weight reduction, and right ventricular function. Int Heart J 2016; 57: 441-8.

13. Den Ruijter HM, Peters SA, Anderson TJ, et al. Common carotid intima-media thickness measurements in cardiovascular risk prediction: a meta-analysis. JAMA 2012; 308: 796-803.

14. Lorenz MW, Polak JF, Kavousi M, et al; PROG-IMT Study Group. Carotid intima-media thickness progression to predict cardiovascular events in the general population (the PROG-IMT collaborative project): a meta-analysis of individual participant data. Lancet 2012; 379: 2053-62.

15. Bartelt A, Bruns OT, Reimer R, et al. Brown adipose tissue activity controls triglyceride clearance. Nat Med 2011; 17: 200-5.

16. Imamura H, Yamaguchi $T$, Nagayama D, Saiki A, Shirai K, Tatsuno I. Resveratrol ameliorates arterial stiffness assessed by cardio-ankle vascular index in patients with type 2 diabetes mellitus. Int Heart J 2017; 58: 577-83.

17. Wang GX, Zhao XY, Meng ZX, et al. The brown fat-enriched secreted factor $\mathrm{Nrg} 4$ preserves metabolic homeostasis through attenuation of hepatic lipogenesis. Nat Med 2014; 20: 1436-43.

18. Jiang J, Lin M, Xu Y, et al. Circulating neuregulin 4 levels are inversely associated with subclinical cardiovascular disease in obese adults. Sci Rep 2016; 6: 36710.

19. Bersell K, Arab S, Haring B, Kühn B. Neuregulin1/ErbB4 signaling induces cardiomyocyte proliferation and repair of heart injury. Cell 2009; 138: 257-70.

20. Rohrbach S, Yan X, Weinberg EO, et al. Neuregulin in cardiac hypertrophy in rats with aortic stenosis. Differential expression of erbB2 and erbB4 receptors. Circulation 1999; 100: 407-12.

21. Iwamoto R, Yamazaki S, Asakura M, et al. Heparin-binding EGF-like growth factor and ErbB signaling is essential for heart function. Proc Natl Acad Sci U S A 2003; 100: 3221-6.

22. Bernard JK, McCann SP, Bhardwaj V, Washington MK, Frey MR. Neuregulin-4 is a survival factor for colon epithelial cells both in culture and in vivo. J Biol Chem 2012; 287: 39850-8. 